\title{
Aprendizaje y conocimiento conectivista para la Educación del siglo XXI
}

\author{
CONNECTIVE KNOWLEDGE AND LEARNING FOR EDUCATION IN THE \\ TWENTY-FIRST CENTURY
}

\author{
Luis Fernando Ibañez Acosta ${ }^{1}$
}

\section{RESUMEN}

El presente estudio de tipo documental explora el surgimiento del conectivismo en la era digital. Por medio de la presentación introductoria de los teóricos de la educación como George Siemens y Stephen Downes, se busca fundamentar una nueva teoría del aprendizaje, sustentada en la epistemología del conocimiento conectivo. La educación del siglo XXI, sacudida por la tecnología digital, solicita una urgente revisión crítica de las clásicas teorías del aprendizaje como el conductismo, el cognitivismo y el constructivismo. El conectivismo, como innovación teórica emergente, busca responder a las necesidades del ámbito del aprendizaje de los nativos digitales y la práctica docente en la actualidad.

Palabras clave: era digital, pensamiento en red, conocimiento conectivista, aprendizaje conectivista.

' Doctorando en Educación. Universidad Iberoamericana (UNIBE). Convenio Programa Pablo Neruda de la OEI y la Red Iberoamericana de Doctorados en Educación-RIDE-. Máster en Comunicación e Información por la Universidade Federal do Rio Grande do Sul (UFRGS). Especialista en Comunicación por la Universidade do Vale do Rio dos Sinos (UNISINOS), Sao Leopoldo, Brasil. Lic. en Filosofía por la Universidad Católica Ntra. Sra. de la Asunción (UCA). Docente en la Universidad Católica de Asunción. 


\begin{abstract}
This research study explores the rise of connectivism in a digital era. Through an introduction to educational theorists George Siemens and Stephen Downes, this work aims at sustaining a new learning theory based on the epistemology of connective knowledge. Education in the twenty-first century is shaken by digital technology, and it compells a critical revision of classical learning theories such as conductivism, cognitivism, and constructionism. As an emergent theoretical innovation, connectivism seeks answers to the needs of digital native learners and current teaching practices.
\end{abstract}

Keywords: digital eral, network thinking, connective knowledge , connective learning. 


\section{INTRODUCCIÓN}

No cabe dudas que ya estamos viviendo en un contexto histórico donde las formas de pensar, conocer, trabajar, enseñar y aprender, entre otros aspectos, están sufriendo cambios en un proceso acelerado de mutación, generado fundamentalmente por la incorporación, desarrollo y consolidación de las nuevas tecnologías de la información y comunicación. Basta simplemente observar, a modo de ilustración, la danza de conceptos como "Simios Informatizados", "Homo informaticus", "Inmigrantes y Nativos Digitales", "Generación Net" , "Aprendizaje 2.0", etc.

En la búsqueda de responder a las demandas del fenómeno educativo actual, acorralado por las novedades tecnológicas, existe la imperiosa necesidad de actualizar la acción formadora institucional para que la misma no quede fuera del circuito del conocimiento en la era digital, caracterizada por la abundancia y el crecimiento exponencial de informaciones. En este mismo orden, está emergiendo una nueva concepción para la educación del siglo XXI, denominada por sus mentores como "conectivismo", que fundamentalmente presenta la innovación en su modo de explicar el conocimiento y el aprendizaje.

El presente trabajo -por tanto- pretende explorar una teoría emergente, como lo constituye la perspectiva conectivista, con implicaciones para la teoría del conocimiento y el aprendizaje. Por de pronto, varias son las preguntas subyacentes que el análisis buscará contestar: ¿Vamos a seguir hablando sin más ni más de teorías clásicas del aprendizaje (conductismo, cognitivismo y constructivismo) en el contexto de la educación del siglo XXI? ¿Está emergiendo otra perspectiva teórica que ya se está presentando como una verdadera innovación en el área de la teoría del aprendizaje?.

\section{TeORía DEL CONOCIMIENTo CONECTIVISTA}

Para comprender la teoría del aprendizaje conectivista, nada mejor que analizar la perspectiva que ofrece Siemens ${ }^{2}$ en su obra "Conociendo el conocimiento" (2006) $)^{3}$.

${ }^{2}$ George Siemens es Fundador y Presidente de Complexive Systems Inc. Actualmente forma parte del Instituto de Investigación del Conocimiento Mejorado por la Tecnología en la Universidad de Athabasca (Canadá). Es un estratega de medios sociales, que se dedica a la planificación, investigación e implementación de las tecnologías sociales de red. También fue Director Asociado de Investigación y Desarrollo con el Centro de Tecnologías del Aprendizaje en la Universidad de Manitoba. Además, es conferencista internacional sobre los nuevos medios del aprendizaje, el cambio sistémico, los medios sociales y el aprendizaje así como del conectivismo.

${ }^{3}$ La obra Knowing Knowledge (Conociendo el Conocimiento) fue escrita por Siemens en el año 2006. Su traducción estuvo a cargo del grupo Nodos Ele en el 2010. Es un libro 
Según el autor, su texto es una "exploración" de las nuevas visiones teóricas del conocimiento y el aprendizaje en el contexto actual de cambios, vinculados tanto al entorno virtual como físico. Advierte, además, que a pesar de dirigir con fuerza su interés hacia el análisis del entorno de la educación virtual, su interés se dirige también al fenómeno físico de la Educación.

Los cambios de que trata este libro no solo tienen relación con entornos tecnológicos o digitales. Hace falta un reordenamiento de los espacios físicos de escuelas y organizaciones. La existencia de condiciones adecuadas para un eficaz flujo de conocimiento, así como de óptimas oportunidades para conectar, recombinar y recrear, es un tema de vital importancia, tanto en la red como en las estructuras y espacios físicos (Siemens, 2006: 143).

Evidentemente esta aclaración es fundamental, ya que está poniendo énfasis en que el conectivismo, como nueva teoría del aprendizaje, no es simplemente un constructo para la enseñanza-aprendizaje en línea en contexto de las nuevas tecnologías de la comunicación e información, sino también apunta a cuestionar y ofrecer una alternativa para el entorno de la Educación Tradicional.

Cuando Stephen Downes y yo dimos un curso sobre conectivismo, sobre conocimiento conectado, en 2008, la conversación se dio con la opinión de personas muertas en la red de aprendizaje, lo que es sin duda un asunto algo extremo. Pero el punto es que leemos teorías y autores, leemos a Shakespeare, a Aristóteles, a filósofos de hace cien años o incluso de hace 30 y no importa si viven o no: podemos conectarnos con sus ideas (Siemens, 2004: 44).

\section{El mundo cambiante y las características del conocimiento}

Siemens (2004), en su diagnóstico de la situación del conocimiento, escribe que el mismo está teniendo una vigencia muy reducida, ya que la información crece exponencialmente. La vida útil del conocimiento, desde una perspectiva tradicional, es considerada como algo inmutable, $\sin$ fecha de vencimiento; sin embargo, el autor señala que hoy el criterio para medir la vigencia de un conocimiento, está en observar simplemente el momento en que fue adquirido y el momento en que

electrónico con licencia Creative Commons, disponible en: http://www.nodosele.com/ editorial.

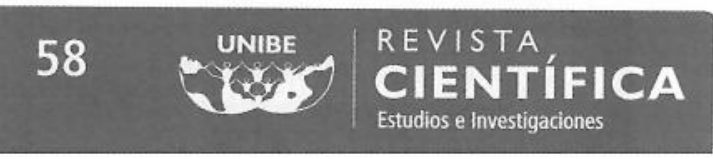


perdió vigencia. Hace 40 años, según el autor, los aprendices al terminar su carrera, tenían la garantía de que sus conocimientos les servirían para toda la vida.

La mitad de lo que es conocido hoy, no era conocido hace 10 años. La cantidad de conocimiento en el mundo se ha duplicado en los últimos 10 años y se duplica cada 18 meses de acuerdo con la Sociedad Americana de Entrenamiento y Documentación (ASTD, por sus siglas en inglés). Para combatir la reducción en la vida media del conocimiento, las organizaciones han sido obligadas a desarrollar nuevos métodos para llevar a cabo la capacitación (Siemens, 2004: 1).

Pero veamos con más detenimiento, las características del conocimiento en el mundo actual, según Siemens:

En primer lugar, al explorar sobre la situación del conocimiento, Siemens constata que estamos ante "un mundo de conocimiento cambiante", caracterizado por los siguientes elementos: cambios en el modo cómo verificamos y validamos el conocimiento; de cómo expresamos y difundimos nuestras ideas; el tipo de lectura que se está dando teniendo en cuenta la emergencia del periódico online, etc. El autor señala, que estamos pasando del consumo de medios masivos a los medios del conocimiento.

Los signos del cambio con las consecuencias generadas por la "liberación del conocimiento", van a impactar sobre todo o ya están impactando, en el modo cómo nos organizamos, ya sea a nivel educativo, religioso, laboral, político, etc. Es decir, las relaciones tradicionales de control/ monitorización y causa/efecto darán paso a otras, caracterizadas por experiencias dinámicas, adaptadas y personalizadas.

En tercer lugar, estamos ante la vigencia de "dos mundos" educativos ${ }^{4}$. En efecto, persiste con fuerza la Educación Tradicional, correspondiente a la era industrial y, la otra, que está en proceso de configuración, denominada Educación Virtual. De todos modos, el autor indica que la educación del pasado no cederá fácilmente su lugar a la educación del mañana.

¿Por qué tantas cosas de la sociedad actual se parecen tanto a las del pasado? Las escuelas, el gobierno, las organizaciones religiosas, los medios de comunicación, han ganado en complejidad, pero han

${ }^{4}$ Esta forma de análisis, se encuentra también en el trabajo de Roberto Aparici, que en un artículo titulado «Conectividad en el Ciberespacio», publicado en 2010, hace la diferenciación entre la educación tradicional, que sigue desconectada de la realidad digital, con una práctica de enseñanza transmisiva y reproductora, originada en contexto de la sociedad industrial. 
mantenido su estructura general y su forma. La estructura de un aula hoy en día, a excepción de un ordenador o un proyector, permanece particularmente inalterada: el profesor de frente, los estudiantes en filas... Lo que hemos hecho básicamente es transferir (no transformar) nuestra identidad física a los espacios y estructuras online (Siemens, 2006: 16).

Profundizando el diagnóstico rápido que realiza Siemens en su sugerente texto, manifiesta igualmente que tanto la comunicación y educación, tal como las conocemos, continúan arraigados en un modelo unidireccional, donde prima la estructura configurada jerárquicamente. Este tipo de reflexión se acerca bastante a lo que el filósofo de la educación latinoamericana, Paulo Freire, en otro contexto, previo al advenimiento de esta era, pregonaba la necesidad de la implementación de una educación problematizadora para propiciar la liberación de los pueblos oprimidos, según Foresti $(2012)^{5}$.

Los medios de comunicación y la educación han sido diseñados en gran parte sobre un modelo de flujo unidireccional (estructura impuesta jerárquicamente). Las jerarquías, a diferencia de las redes y las ecologías, no permiten una rápida adaptación a las tendencias ajenas a su estructura establecida. La estructura es creada por unos pocos elegidos e impuesta sobre la mayoría. Los periódicos publican, nosotros consumimos. El profesor enseña, nosotros aprendemos. Las noticias se emite, nosotros escuchamos (Siemens, 2006: 18).

Si la situación citada todavía es muy fuerte en la sociedad actual, sin embargo, por medio de la tecnología, se está superando cada día más el modelo unidireccional, sobre todo por medio de la participación de los usuarios, quiénes están utilizando herramientas simples y sociales, como lo blogs, wikis, podcasting, videocasting, que a su vez, facilitan nuevas conexiones y permiten la retroalimentación.

${ }^{5}$ En realidad el texto "Proposta de um conceito de aprendizagem para a era digital" de los autores Andresa Foresti y Adriano Cabanabarro Teixeira, de la Universidade de Paso Fundo, Rio Grande do Sul, Brasil, enfoca el análisis comparativo de tres autores: Paulo Freire, George Siemens y Seymour Papers, siendo este último autor , conocido por haber introducido el proyecto de una computadora por niño, que actualmente se está implementando en varios países, y que incluso en Paraguay, aunque no se masificó todavía, sin embargo ya se está implementando el uso de las laptops XO, en el Departamento de la Cordillera. Los tres autores trabajan conceptos como interacción, diálogo, colaboración y creatividad.

\section{0} UNIBE |REVISTA 
Desde una perspectiva de antropología filosófica, expresa que el conocimiento debe ser enfocado desde la visión holística ${ }^{6}$, para superar el reduccionismo, y así dar pasos hacia la interrelación entre los aspectos cognitivos, emocional, físico y espiritual. Esta comprensión del conocimiento está vinculada fundamentalmente a la interconexión humana, ya que "La vida no se vive en un silo". Insiste -desde su interés de humanizar el conocimiento- que el mismo nos llega a través de "una red de prejuicios, opiniones, intervenciones, autocorrecciones, presunciones y exageraciones" (Siemens: 2006, p.10)

En este sentido, enfatiza que el conocimiento debe tener en cuenta los diversos saberes, como: el saber sobre ( eventos, conceptos, etc.); saber hacer ( realizar una actividad, practicar una profesión); saber ser ( expresar conocimiento con humanidad); saber dónde (buscar datos en archivos, bibliotecas, internet, etc.); por último, saber transformar ( innovar, pensar, aplicar). Siemens, advierte que no siempre estos saberes van juntos, sino más bien, aparecen fragmentados.

Tanto en la sociedad actual como en las instituciones educativas, se ponen más énfasis en el saber sobre y saber hacer, dejándose de lado el saber ser, saber dónde y saber transformar, que son fundamentales para vivir en el contexto de la sociedad del conocimiento.

Todavía no hemos integrado en las estructuras educativas las habilidades y procesos que harán de nosotros ciudadanos del mañana. Mientras algunos están trabajando con estas nuevas perspectivas, la gran mayoría se refugia en las estructuras, preparando a estudiantes y trabajadores para un futuro que nunca existirá (Siemens, 2006: 10).

\section{Las nuevas tendencias del conocimiento}

La teoría del aprendizaje de Siemens, se basa en un análisis pormenorizado de los diversos cambios que se están dando en el ecosistema donde se desarrolla el conocimiento ${ }^{7}$.

${ }^{6}$ La visión holística ( analizar el conjunto) está relacionada con perspectiva que hace crítica a la visión surgida en la modernidad (siglos XVII, XVIII, XIX), a instancias del pensamiento del filósofo y físico Isaac Newton, en la física y astronomía y, del filósofo René Descartes, que introdujo la dicotomía entre la mente y el cuerpo. Ambos pensadores son considerados como precursores de la corriente mecanicista del análisis de la naturaleza y la persona. Con el pensamiento postmoderno, y de la mano de la física moderna, la biología molecular, y la neurociencia, aparece el cambio de paradigma, para introducir categorías como sistema, totalidad, ecología, relación psicosomática, entre otras.

${ }^{7}$ Véase el desarrollo de este apartado, en forma extensa, en Siemens (2006) desde la pág. 72 al 78. 
El autor observa, en primer lugar, que se está dando un "ascenso del individuo" que quiere crear, controlar y conectarse más, en contraste con otras épocas. Pretende convertirse en cocreador y no simplemente en consumidor del conocimiento.

Por otro lado, la persona va forjando una nueva "identidad" a través de su participación en la red, donde -por ejemplo- por medio del uso de un blog, podcast u otros recursos, está esparciendo su pensamiento y vida, quedando al descubierto de otras personas, tanto por lo que dice como por lo que hace.

En tercer lugar, la capacidad de "conectividad" ayuda a la adaptación. La cantidad de conexiones demuestran la capacidad de una persona u organización para adaptarse, por un lado, y por otro, poder crear y distribuir sus propios materiales. Estamos pasando del conocimiento en entidades físicas (libros, bibliotecas, etc.) al conocimiento digital, que posibilita la operación de enlazar una idea con otra, un texto con otro.

Todo se integra con todo. Los biólogos usan el lenguaje y los conceptos de los físicos. Los psicólogos utilizan el lenguaje de los neurólogos. Los descubrimientos en un dominio se desplazan al modo de las olas a través de las redes del conocimiento humano. Una vez derribadas las puertas de una disciplina, aparecen los pasillos que otros estaban buscando (Siemens, 2006: 73).

Encuentra además, que el conocimiento actualmente, está relacionado con la importancia del conducto más que el contenido, en la conexión más que el consumo. Más allá de los contenidos relacionados con libros, artículos, archivos de sonidos y videos -como entendemos comúnmente- las conexiones posibilitan llegar a otros contenidos y personas, independientemente del lugar y momento.

Un contenido es conocimiento congelado en un determinado momento (un artículo de revista), mientras que una conexión es un oleoducto para que siga fluyendo nuevo conocimiento (Siemens, 2006: 76).

La idea del conocimiento como certeza está cambiando. La duda es un componente importante del conocimiento. El binomio seguridad/certeza está en suspenso. Puede ser que en el momento presente exista la certeza, pero no sabemos mañana.

Nuestra habilidad para aprender lo que vamos a necesitar mañana, es más importante que lo que sabemos hoy, cuando se necesita conocimiento, pero no se dispone de él, la habilidad para conectarse

\section{UNIBE REVISTA}


a fuentes que satisfagan esas demandas se convierte en decisiva (Siemens, 2006: 83).

El conocimiento está siendo representado por la mediación mediática: texto, audio, video, juegos y simulaciones están reflejando ideas, conceptos y emociones. La representación mediática, cada día más está configurando la vida de la gente. Así, teléfonos móviles con cámaras, espacios sociales en línea, entre otros, están "archivando nuestras vidas", según Siemens.

\section{La base epistemológica de su teoría}

La teoría del conocimiento de Siemens, está basada en el estudio del pensador canadiense sobre nuevas tecnologías y el aprendizaje, Stephen Downes ${ }^{8}$, quién en 2005 publicó el texto "Una introducción al conocimiento conectivo".

En la obra, el autor canadiense advierte que el conectivismo es una "forma de conocimiento nueva", que surge evidentemente de la "interacción entre entidades conectivistas". De este modo, salta del hecho de considerar a una sola identidad como poseedora del conocimiento, para enfatizar que lo que se está dando en la actualidad, a instancias de la tecnología, es la distribución del conocimiento, que se encuentra evidentemente, además de las mentes de las personas, en los artefactos tecnoculturales como los ordenadores y la red internet.

Este hecho, según Downes, pone en crisis al sistema educativo tradicional y a los agentes que se dedican a difundir conocimientos, ya que todavía defienden la postura de que son casi los únicos lugares donde se pueden buscar y en encontrar el conocimiento; sin embargo, el ciberespacio se está constituyendo ya como un lugar privilegiado para "compartir conocimientos". Es decir, el conocimiento está descentralizado y distribuido, tanto en personas, artefactos y redes.

${ }^{8}$ Downes, Stephen. Conociendo el conocimiento, diciembre, 2005. Traducción de Diego Leal (2007). Otra obra importante que ayuda a comprender la propuesta del autor "El futuro del aprendizaje en línea" (2008) y traducido al español también por Leal (2010). Además, "El buntine Oration: Redes de aprendizaje" (2004). El autor, Canadiense, es investigador del Consejo Nacional de Investigación de Canadá. Es especialista en los campos del aprendizaje en línea, nuevos medios, pedagogía y filosofía. Introdujo el concepto de e-learning 2.0 y junto a George Siemens, desarrolló y definió el concepto de "conectivismo". 


\section{Conectivismo: DEL APRENDIZAJE NATURAL AL TECNOLÓGICo}

La concepción del conectivismo de Siemens se refiere no solamente al aspecto del fenómeno que surge con la sociedad red en contexto de creciente desarrollo de las Tics. El conectivismo -según el autor también encuentra su razón de ser en el mismo espacio natural de interrelación donde las personas se vinculan, es decir, tiene que ver con el proceso básico de socialización ${ }^{9}$.

Es más, el conectivismo es un fenómeno que ocurre en nuestro propio cerebro, ya que algunas ideas que tenemos en nuestra cabeza están funcionando en forma de redes distribuidas. Por tanto, la teoría conectivista se fundamenta en la "forma natural de aprendizaje", tanto en su aspecto de socialización como en el desarrollo en redes neuronales ${ }^{10}$. Desde una perspectiva semejante, Abadí (2007), en un excelente trabajo de carácter antropológico, desarrolla en forma amplia, las implicaciones del "Pensamiento en Red", como la trama de la vida en el contexto de irrupción del ciberespacio. El desafío -para la autora- está en saber entrelazar la red tecnológica con la red humana, para desarrollar la creatividad personal y colectiva.

El poder de detentar el conocimiento se ha diluido; a partir de esta masificación de la información, el verdadero diferenciador es ahora la capacidad de los individuos de generar links originales e inéditos ( Abadí, 2007: 6/93).

Actualmente aprendemos y conocemos a través de la participación en redes, que pueden ser sociales o tecnológicas. Es más, afirma que -ciertas tareas- no podrían ser desarrolladas en forma individual; consecuentemente, deben ser fomentadas las habilidades "socialmente conectadas".

Ilustrando esto, para ser capaz de realizar cualquier tarea compleja, como construir un avión o inclusive reparar un automóvil, se requiere cierto nivel de "especialización conectiva", ya que la diversidad de elementos involucrados impiden que una sola persona desarrolle la labor por sí misma: hay tanta información, que no es posible que la maneja un individuo y debe trabajarla de manera socialmente conectada $^{11}$.

${ }^{9}$ Entrevista realizada a George Siemens, a cargo de Vadillo, Guadalupe. Revista Mexicana a Distancia $\mathrm{N}^{\circ} 6$, agosto, 2011.

${ }^{10}$ Ibíd, p. 42

${ }^{11}$ Ibíd, p. 41.

\section{UNIBE $\mid$ REVISTA


Para demostrar esta idea de cómo se produce el aprendizaje natural en red, el autor recurre a una experiencia personal, que le pasó en su ciudad en México.

Como decía, nací en Cuauhtémoc, en un área cercana a la Ciudad de Chihuahua, no he ido en alrededor de diez años pero solía ir porque tengo familiares allá. Mi punto es que inclusive en ese escenario nosotros aprendíamos el uno del otro a través de nuestras redes. Por lo tanto, al ser un pueblo pequeño, una comunidad agrícola apartada, si no sabías cómo arreglar tu tractor, por ejemplo, tenías que recurrir a alguien en el pueblo, que supiera cómo hacerlo y en algunos casos asociabas tu conocimiento con el del otro. En ese sentido me parece que no existe realmente una barrera frente al Conectivismo porque pienso que es una forma natural de aprendizaje ${ }^{12}$.

\section{TeOría DEL APRENDIZAJE CONECTIVISTA}

Siemens (2004), presenta su teoría del aprendizaje conectivista como la apropiada para dar respuestas al contexto cambiante y las características del conocimiento en el entorno actual. En este sentido manifiesta, y aquí creo está la novedad, que teorías clásicas del aprendizaje como el conductismo, cognitivismo y constructivos, a pesar de sus aportes en sus respectivos tiempos y espacios, sin embargo, en ambiente de fuerte mediación tecnológica, se encuentran limitadas.

Para Siemens, las teorías mencionadas ya no responden a la realidad actual, porque hacen recaer todo el peso del aprendizaje en el individuo, que en la época de un flujo lento del conocimiento tuvieron su razón de ser; sin embargo, actualmente, una persona no puede procesar toda la avalancha de información.

Una visión constructivista del aprendizaje, por ejemplo, sugiere que procesemos, interpretemos y extraigamos significado personal de diferentes formatos de información. ¿Qué sucede, sin embargo, cuando el conocimiento es más un diluvio que un goteo? ¿Qué ocurre cuando el conocimiento fluye demasiado rápido para su procesamiento o interpretación? (Siemens, 2006:33).

Profundizando el tema, encontramos la apreciación de que en el contexto actual el aprendizaje ya no ocurre solamente dentro de una persona (aprendizaje interno) sino que se está dando también por fuera

${ }^{12}$ Ibíd, p. 42 
de la misma (aprendizaje externo), es decir, un aprendizaje almacenado y manipulado por la tecnología.

La inclusión de la tecnología y la identificación de conexiones como actividades de aprendizaje, empieza a mover a las teorías de aprendizaje hacia la edad digital. Ya no es posible experimentar y adquirir personalmente el aprendizaje que necesitamos para actuar (Siemens, 2004:4).

Por tanto, en medio de este tipo de realidad, el conectivismo propone que el aprendizaje se debe dar en un marco de conexiones, donde una persona debe crear su red de nodos de confianza, compuesta de la gente, el contenido y la tecnología, para obtener el conocimiento que necesita en un momento dado. Aquí, "el acto de conocer se ha descargado en la propia red".

Ahora bien, cuáles son las ideas que forman parte del marco teórico del pensamiento de Siemens para construir su discurso sobre la teoría del aprendizaje para la era digital? El autor, en su artículo "¿Q Qué tiene de original el conectivismo?"13, explica que las ideas tanto de filósofos como de teóricos de generaciones anteriores, inspiraron la construcción de su teoría. Por ejemplo, de Vigotsky, extrae el enfoque sociocultural, expresando que las herramientas ayudan a interactuar y hacer cosas; asimila además, el aporte de la teoría del aprendizaje social, a través de Bandura, Bruner, Vigotsky; naturaleza contextual/situacionado del aprendizaje elaborado por Lave y Wenger; conocimiento conectivo de Stephen Downes; concepto de la mente provenientes de las neurociencias, inteligencia artificial, que aportan la idea de que el conocimiento está distribuido sobre numerosos instrumentos, relaciones y artefactos; asimila también conceptos de teóricos de los nuevos medios de comunicación, citando a McLuhan, como figura relevante de la visión del impacto de la tecnología sobre el ser humano. Además, el pensamiento de Siemens, está relacionado con la teoría de la complejidad y la teoría de la red.

En síntesis, fundamentalmente el conectivismo "encuentra sus raíces" en el contexto caracterizado por la abundancia, acelerados cambios, varias fuentes de información y perspectivas; a su vez, todo ello exige saber filtrar y encontrar sentido al caos. Es de destacar que el conectivismo trata los principios del aprendizaje en varios niveles, tales como: biológico/ neuronal, conceptual, social/externo.

13 Siemens, George. Qué tiene de original el conectivismo?. En: http:// humanismoyconectividad.wordpress.com. 6 de agosto, 2008.

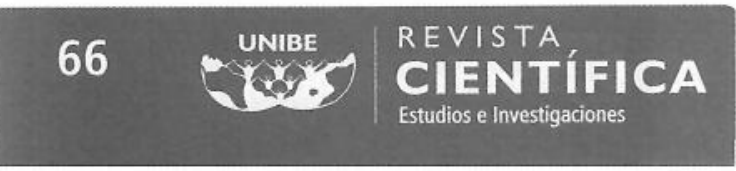




\section{CONCLUSIÓN}

El conectivismo como un objeto de estudio nuevo en el campo de la teoría del aprendizaje, demanda la atención tanto de los teóricos como de los educadores. Además, observando el ámbito del hacer educacional y comunicacional, se constata la necesidad de la implementación de estudios empíricos, para contrastar los principios fundamentales del conectivismo con las realidades emergentes de la sociedad del siglo XXI.

En este sentido, es un estimulo para la realización de estudios en el ámbito académico y para trabajar un marco teórico nuevo en el contexto educacional como el comunicacional.

Por tanto, las contribuciones de George Siemens y Stephen Downes, han abierto un camino a la creatividad intelectual, al proponer la teoría conectivista como un aporte para la renovación de la mirada sobre las teorías clásicas del aprendizaje como el conductismo, cognitivismo y constructivismo, que surgieron en entornos sin la influencia de la tecnología digital. 


\section{BIBLIOGRAFÍA}

ABADÍ, Sonia. (2007). Pensamiento en Red: una nueva disciplina para crear, realizar y vivir. Buenos Aires: Editorial Temas.

APARICI, Roberto. (2010).Conectados en el ciberespacio. Madrid: Editorial UNED.

DOWNES, Stephen. ( 2005). Una introducción al conocimiento conectivo. Traducción de Diego Leal (2007), <http://conectivism. blogspot.com/2012/06/leer-una-introduccion-al-conocimiento.html> [Consulta: agosto 2014]

DOWNES, Stephen.(2006). Redes de aprendizaje y conocimiento conectivo. <http://juandomingofarnos.wordpress.com > [ Consulta: agosto 2014]

FORESTI, Andrea y CABANABARRO, Teixeira. (2012). Proposta de um conceito de aprendizagem para a era digital. Revista Latinoamericana de Tecnología Educativa. Vol. 11, $\mathrm{n}^{\circ}$ 2. <http://campusvirtual.unex.es/ revistas/index.php/relatec/article/view/919 >[Consulta: agosto 2014]

SIEMENS, George. (2006). Conociendo el conocimiento. Traducido por el Grupo Nodos Ele en 2010, < http://www.nodosele.com/editorial> [Consulta: nov.2013]

SIEMENS, George. (2004). "Conectivismo: una teoría del aprendizaje para la era digital". Traducido por Diego Fonseca (2007).Publicado bajo una Licencia Creative Commons 2.5. <http//fernandosantamaria. $\mathrm{com} / \mathrm{blog} / 2006 / 09 /$ conectivismo-una-teoria-de-aprendizaje-para-la-eradigital-ii/ > [Consulta: nov. 2013]

SIEMENS, George.(2008). Qué tiene de original el conectivismo?. $<$ http://humanismoyconectividad.wordpress.com/2009/01/14/ conectivismo-siemens/ > [Consulta: agosto: 2014]

VADILLO, Guadalupe. Entrevista a George Siemens, desarrollador del conectivismo. Revista Mexicana a Distancia No $\mathrm{N}^{\circ}$, agosto, 2011. < http:// bdistancia.ecoesad.org. $\mathrm{mx} /$ ?articulo=entrevista-a-goerge-siemensdesarrollador-del-conectivismo $>$ [Consulta agosto.2014]

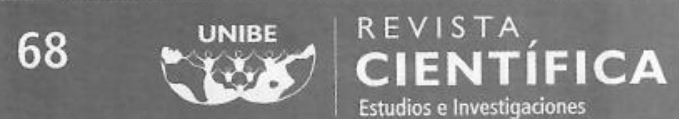

